\title{
Open inflation models and gravitational wave anisotropies in the CMB
}

\author{
Juan García-Bellido \\ Theory Division, CERN, CH-1211 Geneva 23, Switzerland
}

(Received 27 February 1997)

\begin{abstract}
We study the large scale power spectrum of gravitational wave perturbations of the microwave background in the context of single-bubble open inflation models. We compute the ratio of tensor to scalar contributions to the CMB anisotropies as a function of $\Omega_{0}$, the spectral index $n_{S}$, and the tunneling parameter $2 \pi G S_{1} / H$. We find that gravitational wave anisotropies can be very large at small values of this tunneling parameter. We also consider the contribution of supercurvature and bubble wall modes and find constraints on the parameters of open inflation models from the observed temperature anisotropies of the CMB. We show that the induced gravity and open hybrid scenarios are compatible with present observations for a reasonable range of parameters. [S0556-2821(97)04818-2]

PACS number(s): $98.80 . \mathrm{Cq}$
\end{abstract}

\section{INTRODUCTION}

The inflationary paradigm [1] not only gives an explanation of the large scale homogeneity and isotropy of our observable Universe, but also predicts an almost scale-invariant spectrum of primordial metric perturbations that could be responsible for the observed temperature anisotropies of the cosmic microwave background (CMB) as well as the origin of large scale structure [2].

Until recently, observations of the CMB temperature anisotropies provided just a few constraints on the parameters of inflationary models, mainly from the amplitude and the tilt of both scalar and tensor perturbations' spectrum [3]. Nowadays, with a dozen experiments looking at different angular scales, we have much more information about the primordial spectra as well as other cosmological parameters such as $\Omega_{0}, H_{0}, \Omega_{\mathrm{B}}$, etc; see, e.g., [4]. In the near future, high resolution observations of the microwave background anisotropies with the recently approved satellites, the Microwave Anisotropy Probe (MAP) [5] and PLANCK [6], will determine the cosmological parameters and the main features of the primordial spectra of density and gravitational wave perturbations with better than $1 \%$ accuracy [7-9]. This means that cosmology is becoming a phenomenological science, where observations or experiments determine parameters and allow us to test alternative models of the Universe. A special effort is therefore needed from theoretical cosmologists in order to predict the essential features as well as possible variations of the expected CMB power spectrum of temperature anisotropies. There has been tremendous progress in this direction in the last few years and will probably increase as we approach the time in which the satellites will be launched.

Inflation has generically been associated with a flat universe, due to its tendency to drive the spatial curvature so effectively to zero. However, it is now understood that inflation comprises a wider class of models, some of which may give rise to an open universe at present [10-13]. Such models generically contain a field trapped in a false vacuum, which tunnels to its true vacuum via the nucleation of a single bubble, inside which a second period of inflation drives the universe to almost flatness. The original motiva- tion of open inflation as a model where one could reconcile a large value of the Hubble constant [14] with the large estimated age of globular clusters [15] is no longer essential due to the recent recalibration of distances by the Hipparcos satellite [16], which has brought down both the rate of expansion, $H_{0}=60 \pm 10 \mathrm{~km} \mathrm{~s}^{-1} \mathrm{Mpc}^{-1}$, and the age of the universe, $t_{0}=12 \pm 2 \mathrm{Gyr}$, thus becoming compatible with an Einstein-de Sitter model; see, e.g., [17]. Large scale structure observations, however, seem to be in conflict with a flat universe and $h>0.5$, since they favor low $\Omega_{0} h$; see, e.g., [18]. Apart from still uncertain cosmological observations, open inflation models have several interesting theoretical features that single them out from other cosmological models, in particular the way they solve the homogeneity problem independently from the flatness problem [12]. Furthermore, if future observations determine $\Omega_{0}$ to be less than 1 with better than $1 \%$ accuracy, we will have to invoke open inflation models to explain the large scale homogeneity.

In open inflation models the origin of structure is still related to amplified quantum fluctuations of the field that drives inflation inside the bubble $[19,11]$. A distinct feature of these models is that in the spectrum of metric perturbations there appears a discrete supercurvature mode [20,21], associated with the open de Sitter vacuum [19,22], as well as a mode associated with the bubble wall fluctuations at tunneling [12,23-25], whose contribution could be made small in some of the models [26,27]. Furthermore, there is some evidence that the observations made in a wide range of scales, from horizon size to large clusters of galaxies, constrain open inflation models (with small $\Omega_{0} \sim 0.3-0.4$ ) to have a "tilted" spectrum of density perturbations with spectral index $n_{S}>1$ [28,29], and essentially no other contribution, either from gravitational waves or supercurvature modes. In order to account for these observations, we have recently proposed a tilted hybrid model of open inflation [30] and computed the scalar component of the CMB power spectrum. For an alternative way of producing a large tilt see Ref. [31].

Apart from scalar metric perturbations, open inflation also produces a primordial spectrum of gravitational waves, whose amplitude and scale dependence in single-bubble open inflation models has only recently been known [32]. In 
order to compare with observations one has to compute the corresponding angular power spectrum $C_{l}$ of CMB temperature fluctuations [33]. Since the gravitational wave contribution to the power spectrum decays beyond $l \sim 30$, it is enough to consider the large scale (low multipole) tensor power spectrum, where gravitational redshift is the dominant effect, without the need of large computer codes to calculate the full power spectrum. In this paper we will calculate the first ten multipoles of the CMB power spectrum for both scalar and tensor components in an open universe, and determine the ratio of tensor to scalar contributions as a function of $\Omega_{0}$ and other model parameters. We will then constrain general single-bubble open inflation models from such a tensor component of the $\mathrm{CMB}$ anisotropies.

\section{QUANTUM TUNNELING AND SLOW-ROLL INFLATION}

We will concentrate here in the single-bubble open inflation models with two fields, the tunneling field $\sigma$ and the inflaton field $\phi$. The former determines the geometry of the bubble and the latter produces the inhomogeneities in the metric responsible for the observed temperature anisotropies of the microwave background.

The $\sigma$ field is initially trapped in its false $(F)$ vacuum and then tunnels to the true $(T)$ vacuum producing a single bubble. The extremal instanton action corresponds to the $\mathrm{O}(3,1)$ symmetric bubble [34]

$$
\begin{aligned}
S_{B}(R)= & 2 \pi^{2} R^{3} S_{1}+\frac{4 \pi^{2}}{\kappa^{2}}\left[\frac{1}{H_{T}^{2}}\left[\left(1-R^{2} H_{T}^{2}\right)^{3 / 2}-1\right]\right. \\
& \left.-\frac{1}{H_{F}^{2}}\left[\left(1-R^{2} H_{F}^{2}\right)^{3 / 2}-1\right)\right],
\end{aligned}
$$

where we have taken into account the contributions from the wall (first term) and the interior of the bubble (in large brackets). Here $R$ is the radius of the bubble, $\kappa^{2}=8 \pi / M_{\mathrm{Pl}}^{2}$, $H_{F}^{2} \equiv \kappa^{2} U_{F} / 3$, where $U_{F}$ is the energy density in the false vacuum (and similarly for $H_{T}$ in the true vacuum) and

$$
S_{1}=\int_{\sigma_{F}}^{\sigma_{T}} d \sigma\left[2\left(U(\sigma)-U_{F}\right)\right]^{1 / 2}
$$

For the thin wall approximation to be valid we require that the width of the bubble wall, $\Delta R$, be much smaller than its radius of curvature, $\Delta R / R \simeq H_{T}(\Delta \sigma) /\left[2\left(U_{0}-U_{F}\right)\right]^{1 / 2} \ll 1$, where $U_{0}$ is the value of the potential at the maximum. The only requirement is that the barrier between $\sigma_{F}$ and $\sigma_{T}$ be sufficiently high, i.e., $U_{0} \gg \Delta U=U_{F}-U_{T}$.

The radius of curvature of the bubble wall is that for which the bounce action (1) is an extremum. An exact solution [34] can be written in terms of dimensionless parameters $a$ and $b$ :

$$
\begin{gathered}
R H_{T}=\left[1+(a+b)^{2}\right]^{-1 / 2} \equiv\left(1+\Delta^{2}\right)^{-1 / 2}, \\
a \equiv \frac{\Delta U}{3 S_{1} H_{T}}, \quad b \equiv \frac{\kappa^{2} S_{1}}{4 H_{T}} .
\end{gathered}
$$

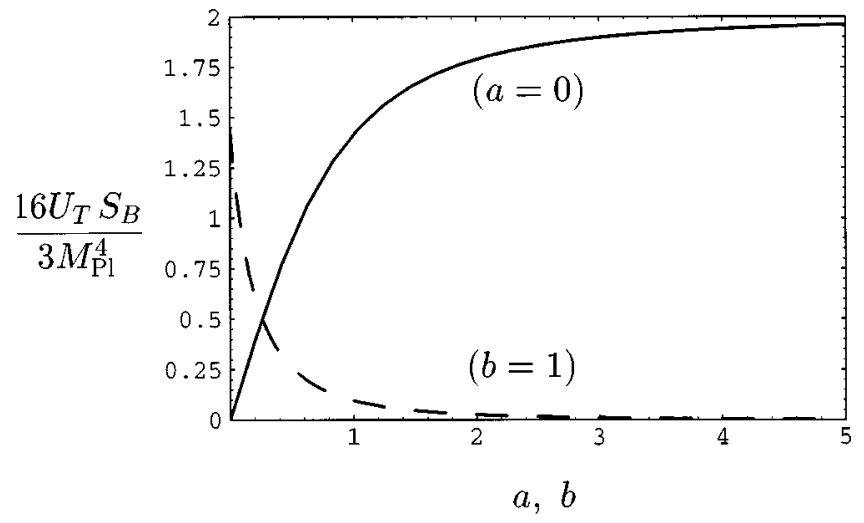

FIG. 1. The bounce action $S_{B}$ as a function of $b$ for $a=0$ (continuous line) and as a function of $a$ for $b=1$ (dashed line), in units of $3 M_{\mathrm{Pl}}^{4} / 16 U_{T}$. It is clear that for a large range of parameters, the bounce action easily satisfies $S_{B} \gg 1$.

Since $S_{1} \sim U_{0} / M \sim M(\Delta \sigma)^{2}$ for a mass $M$ in the false vacuum, the parameter $a \simeq\left(\Delta U / U_{0}\right) M / H_{T}$, which characterizes the degeneracy of the vacua, can be made arbitrarily small by tuning $U_{T} \simeq U_{F}$. On the other hand, the parameter $b \simeq\left(\Delta \sigma / M_{\mathrm{Pl}}\right)^{2} M / H_{T}$, which characterizes the width of the barrier, is not a tunable parameter and could be very large or very small depending on the model. It turns out that the amplitudes of the bubble wall fluctuations and gravitational wave perturbations of the CMB strongly depend on the value of this parameter, as we will discuss in Sec. VI.

In order to prevent collisions with other nucleated bubbles (at least in our past light cone) it is necessary that the probability of tunneling be sufficiently suppressed. For an open universe of $\Omega_{0}>0.2$, this is satisfied as long as the bounce action $S_{B}>6$; see Ref. [10]. This imposes only a very mild constraint on the tunneling parameters $a$ and $b$ (see Fig. 1) as long as the energy density in the true vacuum satisfies $U_{T} \ll M_{\mathrm{Pl}}^{4}$.

Just after tunneling the field $\sigma$ is held in its true vacuum while the field $\phi$ is free to move down its potential, driving a short period of inflation responsible for the approximate flatness of our present universe. The equations of motion of the scalar field during inflation (where we can soon neglect spatial curvature) are

$$
\begin{gathered}
H^{2}\left(1-\frac{\epsilon}{3}\right)=\frac{\kappa^{2}}{3} V(\phi), \\
3 H \dot{\phi}\left(1-\frac{\delta}{3}\right)=-V^{\prime}(\phi),
\end{gathered}
$$

where $\epsilon$ and $\delta$ are defined in terms of the fundamental parameter $H(\phi)$,

$$
\begin{gathered}
\epsilon \equiv-\frac{\dot{H}}{H^{2}}=\frac{2}{\kappa^{2}}\left(\frac{H^{\prime}(\phi)}{H(\phi)}\right)^{2}, \\
\delta \equiv-\frac{\ddot{\phi}}{H \dot{\phi}}=\frac{2}{\kappa^{2}}\left(\frac{H^{\prime \prime}(\phi)}{H(\phi)}\right) .
\end{gathered}
$$

Note that for inflation to occur, we need $\epsilon<1$. The number of $e$-folds of inflation can be computed as 


$$
N(\phi)=\frac{\kappa^{2}}{2} \int \frac{H d \phi}{H^{\prime}(\phi)}=\int \frac{\kappa d \phi}{\sqrt{2 \epsilon}} .
$$

In the slow-roll approximation, we have $\epsilon \ll 1$ and $\delta \simeq \eta-\epsilon \ll 1$, where [2]

$$
\begin{gathered}
\epsilon=\frac{1}{2 \kappa^{2}}\left(\frac{V^{\prime}(\phi)}{V(\phi)}\right)^{2} \ll 1, \\
\eta=\frac{1}{\kappa^{2}}\left(\frac{V^{\prime \prime}(\phi)}{V(\phi)}\right) \ll 1 .
\end{gathered}
$$

All quantities of interest can then be expressed in terms of these slow-roll parameters. In particular, the amplitude and tilt of the primordial spectrum of density and gravitational wave perturbations. We will concentrate on those observables in the following sections.

\section{SCALAR AND TENSOR METRIC PERTURBATIONS}

We briefly review here the theory of gauge invariant scalar and tensor metric perturbations. The most general linearly perturbed metric can be written as [35]

$$
\begin{aligned}
d s^{2}= & a^{2}(\eta)\left[-(1+2 A) d \eta^{2}+2 B_{\mid i} d x^{i} d \eta+\left\{(1+2 \mathcal{R}) \gamma_{i j}\right.\right. \\
& \left.\left.+2 E_{\mid i j}+2 h_{i j}\right\} d x^{i} d x^{j}\right],
\end{aligned}
$$

where $\{i, j\}$ label the three-dimensional open space coordinates with metric $\gamma_{i j}$. The gauge-invariant tensor perturbation $h_{i j}$ corresponds to a transverse traceless gravitational wave, $\nabla^{i} h_{i j}=h_{i}{ }^{i}=0$. The four scalar perturbations are not gauge independent. Under a gauge transformation $\tilde{\eta}=\eta$ $+\xi^{0}\left(\eta, x^{k}\right), \quad \tilde{x}^{i}=x^{i}+\gamma^{i j} \xi_{\mid j}\left(\eta, x^{k}\right)$, they transform as

$$
\begin{gathered}
\widetilde{A}=A-\xi^{0 \prime}-\frac{a^{\prime}}{a} \xi^{0}, \quad \widetilde{\mathcal{R}}=\mathcal{R}-\frac{a^{\prime}}{a} \xi^{0}, \\
\widetilde{B}=B+\xi^{0}-\xi^{\prime}, \quad \widetilde{E}=E-\xi, \\
\widetilde{h}_{i j}=h_{i j},
\end{gathered}
$$

where a prime denotes derivative with respect to conformal time $\eta$. There are, however, only two gauge-invariant gravitational potentials,

$$
\begin{gathered}
\Phi=A+\frac{1}{a}\left[a\left(B-E^{\prime}\right)\right]^{\prime}, \\
\Psi=\mathcal{R}+\frac{a^{\prime}}{a}\left(B-E^{\prime}\right),
\end{gathered}
$$

which are related through the perturbed Einstein equations

$$
\begin{gathered}
\Phi+\Psi=0, \\
2 \frac{k^{2}-3 K}{a^{2}} \Psi=\kappa^{2} \delta \rho .
\end{gathered}
$$

Here $\delta \rho$ is the gauge-invariant density perturbation [35].
In linear perturbation theory, the scalar metric perturbations can be separated into $A\left(\eta, x^{i}\right) \equiv A(\eta) Q\left(x^{i}\right)$, where $Q\left(x^{i}\right)$ are the scalar harmonics, eigenfunctions of the Laplacian:

$$
\nabla^{2} Q(r, \theta, \phi)=-k^{2} Q(r, \theta, \phi) .
$$

These solutions have the general form [36]

$$
Q_{k l m}(r, \theta, \phi)=\Pi_{k l}(r) Y_{l m}(\theta, \phi),
$$

where $Y_{l m}$ are the usual spherical harmonics.

Furthermore, the gravitational wave perturbations can also be written as $h_{i j}\left(\eta, x^{k}\right) \equiv h(\eta) Q_{i j}\left(x^{k}\right)$, where $Q_{i j}$ are the transverse traceless tensor harmonics, $k^{i} Q_{i j}=Q_{i}{ }^{i}=0$, satisfying the same equation (20) as the scalar harmonics $[35,36]$. The radial part of the scalar and tensor harmonics in an open universe can be found in Appendix A.

We are interested in the time evolution of these perturbations during the matter era. The gauge-invariant scalar and tensor perturbations satisfy the following equations during this era:

$$
\begin{gathered}
\Phi^{\prime \prime}+3 \frac{a^{\prime}}{a} \Phi^{\prime}-2 K \Phi=0, \\
h^{\prime \prime}+3 \frac{a^{\prime}}{a} h^{\prime}+\left(k^{2}+2 K\right) h=0,
\end{gathered}
$$

where $K=-1$ (0) for an open (flat) universe. Metric perturbations remain constant outside the Hubble scale during inflation and radiation era and start to evolve as soon as they reenter during the matter era, where they create temperature anisotropies on large scales.

\section{TEMPERATURE ANISOTROPIES}

Quantum fluctuations of the inflaton field $\phi$ during inflation produce long-wavelength scalar curvature perturbations and tensor (gravitational wave) perturbations, which leave their imprint in the CMB anisotropies. Open inflation generates three different types of scalar modes: those that cross outside during the second stage of inflation and constitute a continuum of subcurvature modes $[19,11]$, a discrete supercurvature mode [20], associated with the open de Sitter vacuum [22], and a mode associated with the bubble wall fluctuations at tunneling [24,25,37], all of which induce temperature anisotropies in the microwave background. We have already considered the supercurvature and bubble wall mode in previous publications $[27,30]$. We will concentrate here on the continuum of scalar and tensor subcurvature modes.

Metric perturbations give rise to temperature fluctuations when they reenter the horizon, via gravitational redshift. The dominant effect on large scales is known as the Sachs-Wolfe effect [38]. Due to this effect, scalar metric perturbations on the surface of last scattering are responsible for temperature fluctuations in the CMB with amplitude given by $[38,2]$

\footnotetext{
${ }^{1}$ From now on $A, B$, etc., stand for the $\eta$-dependent functions.
} 


$$
\begin{aligned}
\frac{\delta T}{T}(\theta, \phi)= & \frac{1}{3} \Phi(0) Q\left(\eta_{0}, \theta, \phi\right)+2 \int_{0}^{\eta_{0}} d r \Phi^{\prime}(r) \\
& \times Q\left(\eta_{0}-r, \theta, \phi\right),
\end{aligned}
$$

where $\eta_{0}$ is the present conformal time and $\eta_{\mathrm{LSS}} \simeq 0$ corresponds to the last scattering surface. Note that this expression is valid only for adiabatic fluctuations such as those produced by inflation; see Ref. [2]. The first and second terms are called the "intrinsic" and "integrated" SachsWolfe effect, respectively.

Tensor metric perturbations on the last scattering surface also create temperature fluctuations with amplitude [38]

$$
\frac{\delta T}{T}(\theta, \phi)=\int_{0}^{\eta_{0}} d r h^{\prime}(r) Q_{r r}\left(\eta_{0}-r, \theta, \phi\right),
$$

where $Q_{r r}$ is the $r r$ component of the tensor harmonic along the line of sight.

Temperature anisotropies in the cosmic microwave background are usually given in terms of the two-point correlation function or power spectrum $C_{l}$, defined by an expansion in multipole number $l$ :

$$
\left\langle\frac{\delta T}{T}(\hat{\mathbf{n}}) \cdot \frac{\delta T}{T}\left(\hat{\mathbf{n}}^{\prime}\right)\right\rangle_{\hat{\mathbf{n}} \cdot \hat{\mathbf{n}}^{\prime}=\cos \theta}=\sum_{l} \frac{2 l+1}{4 \pi} C_{l} P_{l}(\cos \theta) .
$$

We are mainly interested in the large scale (low multipole number $l$ ) temperature anisotropies since it is there where gravitational waves could become important. After $l \sim 30$, the tensor power spectrum drops down [39] while the density perturbation spectrum increases towards the first acoustic peak; see Ref. [40]. On these large scales the dominant effect is gravitational redshift via the Sachs-Wolfe effect. Fortunately, this effect can be easily computed without the need for a CMB code. I will review how this is done in the case of both flat and open universe power spectra, and find the relation between the scalar and tensor components of the CMB anisotropies.

\section{FLAT UNIVERSE POWER SPECTRA}

In this section we will briefly review the computation of the large angle (low multipole) scalar and tensor power spectra in a flat universe. On those scales $(l<30)$, the dominant contribution comes from the Sachs-Wolfe effect [38]. On smaller scales $(l>30)$, the scalar component gets contributions not only from gravitational perturbations but also from density and velocity fluctuations, which induce a peak in the spectrum on scales associated with the causal size of the universe at last scattering [41]. On the other hand, the tensor component is not coupled to these last sources of perturbations and decays for large multipole numbers.

\section{Scalar modes}

In a flat universe during the matter era, $a(\eta) \propto \eta^{2}$, the growing mode solution of the scalar perturbation equation (22) is $\Phi=(3 / 5) \mathcal{R}=$ const, where $\mathcal{R}$ is the primordial comoving curvature perturbation during inflation [42]. Thus the induced temperature fluctuation on large scales becomes

$$
\frac{\delta T}{T}=\frac{1}{5} \mathcal{R} Q,
$$

where $Q_{k l m}=\sqrt{2 / \pi} k j_{l}(k r) Y_{l m}(\theta, \phi)$ are the flat scalar harmonics, with radial parts given by spherical Bessel functions. The corresponding power spectrum (26) can be written as

$$
C_{l}^{S}=\frac{4 \pi}{25} \int_{0}^{\infty} \frac{d k}{k} \mathcal{P}_{\mathcal{R}}(k) j_{l}^{2}\left(k \eta_{0}\right),
$$

where $\mathcal{P}_{\mathcal{R}}(k)$ is the primordial spectrum of scalar perturbations, defined by

$$
\left\langle\mathcal{R}_{k} \mathcal{R}_{k^{\prime}}\right\rangle=\frac{2 \pi^{2}}{k^{3}} \mathcal{P}_{\mathcal{R}}(k) \delta\left(k-k^{\prime}\right) .
$$

It is possible to compute the primordial scalar spectrum during inflation [2]:

$$
\mathcal{P}_{\mathcal{R}}(k)=\frac{\kappa^{2}}{2}\left(\frac{H_{T}}{2 \pi}\right)^{2} \frac{1}{\epsilon}
$$

in the slow-roll approximation. The corresponding tilt of the scalar spectrum can be defined as

$$
n_{S}-1 \equiv \frac{d \ln \mathcal{P}_{\mathcal{R}}(k)}{d \ln k} \simeq-6 \epsilon+2 \eta
$$

again in the slow-roll approximation; see Eqs. (10) and (11). For a scale-invariant spectrum, $n_{S}=1$, we can integrate Eq. (28) to give

$$
l(l+1) C_{l}^{S}=\frac{2 \pi}{25} \mathcal{P}_{\mathcal{R}}=\text { const. }
$$

As a consequence it is customary to plot the angular power spectrum as $l(l+1) C_{l}$. On the other hand, for $n \neq 1$, the scalar power spectrum is a complicated function of multipole number $l$ (see Refs. [43,2]):

$$
C_{l}^{S}=\frac{2 \pi}{25} \mathcal{P}_{\mathcal{R}} \frac{\Gamma(3 / 2) \Gamma((3-n) / 2) \Gamma(l+(n-1) / 2)}{\Gamma(2-n / 2) \Gamma[l+2-(n-1) / 2]} .
$$

As mentioned above, the Sachs-Wolfe effect is the dominant contribution only for $l \leqslant 30$, and in fact this last formula breaks down even at moderate $l \sim 20$ due to the rise to the first acoustic peak, see Ref. [44].

\section{Tensor modes}

In a flat universe, the growing mode of the tensor metric perturbation equation (23) during the matter era is given by $h(\eta)=h G_{k}(\eta)$, where $h$ is the primordial gravitational wave perturbation during inflation, which remains constant on large scales and thus can be related to that at reentry during the matter era, and

$$
G_{k}(\eta)=3 \frac{j_{1}(k \eta)}{k \eta}
$$

is normalized so that $G_{k}(0)=1$ at the last scattering surface. The induced temperature fluctuation amplitude (25) is then 


$$
\frac{\delta T}{T}=\int_{0}^{\eta_{0}} d r h G_{k}^{\prime}\left(\eta_{0}-r\right) Q_{r r}(r)
$$

where $Q_{r r}$ is the $r r$ component of the flat universe tensor harmonic:

$$
Q_{r r}(r)=\left[\frac{(l-1) l(l+1)(l+2)}{\pi k^{2}}\right]^{1 / 2} \frac{j_{l}(k r)}{r^{2}} .
$$

The corresponding tensor power spectrum can be written as $[45,39]$

$$
C_{l}^{T}=\frac{9 \pi}{4}(l-1) l(l+1)(l+2) \int_{0}^{\infty} \frac{d k}{k} \mathcal{P}_{g}(k) I_{k l}^{2},
$$

where $I_{k l}$ is given by

$$
I_{k l}=\int_{0}^{x_{0}} d x \frac{j_{2}\left(x_{0}-x\right) j_{l}(x)}{\left(x_{0}-x\right) x^{2}},
$$

with $x \equiv k \eta$, and $\mathcal{P}_{g}(k)$ is the primordial spectrum of gravitational wave perturbations, defined by

$$
\left\langle h_{k} h_{k^{\prime}}\right\rangle=\frac{2 \pi^{2}}{k^{3}} \mathcal{P}_{g}(k) \delta\left(k-k^{\prime}\right) .
$$

It is possible to compute the primordial tensor perturbation spectrum during inflation [39,2]:

$$
\mathcal{P}_{g}(k)=8 \kappa^{2}\left(\frac{H_{T}}{2 \pi}\right)^{2} .
$$

The corresponding tilt of the gravitational wave spectrum can be defined as

$$
n_{T} \equiv \frac{d \ln \mathcal{P}_{g}(k)}{d \ln k} \simeq-2 \epsilon
$$

in the slow-roll approximation (10). For a scale-invariant spectrum, $n_{T}=0$, we can integrate Eq. (37) to give [39]

$$
l(l+1) C_{l}^{T}=\frac{\pi}{36}\left(1+\frac{48 \pi^{2}}{385}\right) \mathcal{P}_{g} A_{l},
$$

where $A_{l}=(1.1184,0.8789, \ldots)$ for $l=2,3, \ldots$, which approaches $A_{l}=1$ for large multipoles, $l \sim 30$, and thus $l(l+1) C_{l}^{T}$ becomes constant in that limit. Beyond this value of $l$ the gravitational waves have redshifted away before last scattering and beyond the limits of the Sachs-Wolfe integral. Thus expression (42) is valid only for $l \lesssim 30$; see [39].

We can compute the ratio of tensor to scalar contributions to the $\mathrm{CMB}$ angular power spectrum in a flat universe, using Eqs. (42) and (32) at large $l$ [2]

$$
R \equiv \frac{C_{l}^{T}}{C_{l}^{S}}=\frac{25}{72}\left(1+\frac{48 \pi^{2}}{385}\right) \frac{\mathcal{P}_{g}}{\mathcal{P}_{\mathcal{R}}} \simeq 12.4 \epsilon \simeq 6.2\left|n_{T}\right|,
$$

in the slow-roll approximation. This is the well-known relation between the ratio $R$ and the tilt of the gravitational wave spectrum. In most inflationary models the slow-roll parameter $\epsilon$ is so small that there is essentially no gravitational wave contribution to the power spectrum and it becomes extremely difficult to measure this relation (see Ref. [6]) unless polarization effects are taken into account [46]. Therefore, it is worth considering whether this relation holds in an open universe and how it characterizes different inflationary models.

\section{OPEN UNIVERSE POWER SPECTRA}

In an open universe, $\Omega<1$, there is a characteristic scale associated with spatial curvature, $1 / a^{2}=H^{2}(1-\Omega)$. As a consequence the Hubble scale approaches the curvature scale from below as time progresses, and exceeds it only if there were a nonvanishing cosmological constant. Furthermore, conformal time $\eta=\int d t / a$ can be interpreted as the coordinate distance to the particle horizon. Its present value $\eta_{0}$ is to a very good approximation the distance to the last scattering surface in units of the curvature scale and, assuming that the universe is matter dominated, it is given by

$$
\eta_{0}=\operatorname{arccosh}\left(\frac{2}{\Omega_{0}}-1\right)
$$

For $\Omega_{0}<2 /(1+\cosh 1) \simeq 0.786$, the last scattering surface is located beyond the curvature scale.

As discussed in the previous section, since the gravitational wave contribution to the power spectrum decays beyond $l \sim 30$ (see Ref. [33]) it is enough to consider the large scale (low multipole) tensor power spectrum, where gravitational redshift is the dominant effect. In this section we will calculate the first ten multipoles of the CMB power spectrum for both scalar and tensor components in an open universe, and determine the ratio of tensor to scalar contributions as a function of $\Omega_{0}$ and other model parameters.

\section{Scalar modes}

The growing mode solution to the scalar perturbation equation (22) in an open universe during the matter era, with $a(\eta)=a_{0}(\cosh \eta-1)$, is $\Phi(\eta)=(3 / 5) \mathcal{R} F(\eta)$, where $\mathcal{R}$ is the primordial scalar perturbation after Hubble-scale crossing during inflation and

$$
F(\eta) \equiv 5 \frac{\sinh ^{2} \eta-3 \eta \sinh \eta+4(\cosh \eta-1)}{(\cosh \eta-1)^{3}}
$$

is normalized so that $F(0)=1$ at last scattering. Because of the Sachs-Wolfe effect, the induced temperature fluctuations take the expression (24), where $\eta_{0}$ is given by Eq. (44). The corresponding power spectrum (26) can be written as

$$
C_{l}^{S}=\frac{2 \pi^{2}}{25} \int_{0}^{\infty} \frac{q d q}{1+q^{2}} \mathcal{P}_{\mathcal{R}}(q) I_{q l}^{2}
$$

where $q^{2}=k^{2}-1$, and $I_{q l}$ is given by

$$
q I_{q l}=\Pi_{q l}\left(\eta_{0}\right)+6 \int_{0}^{\eta_{0}} d r \Pi_{q l}(r) F^{\prime}\left(\eta_{0}-r\right) .
$$

Here $\mathcal{P}_{\mathcal{R}}(q)$ is the primordial spectrum of scalar metric perturbations: 


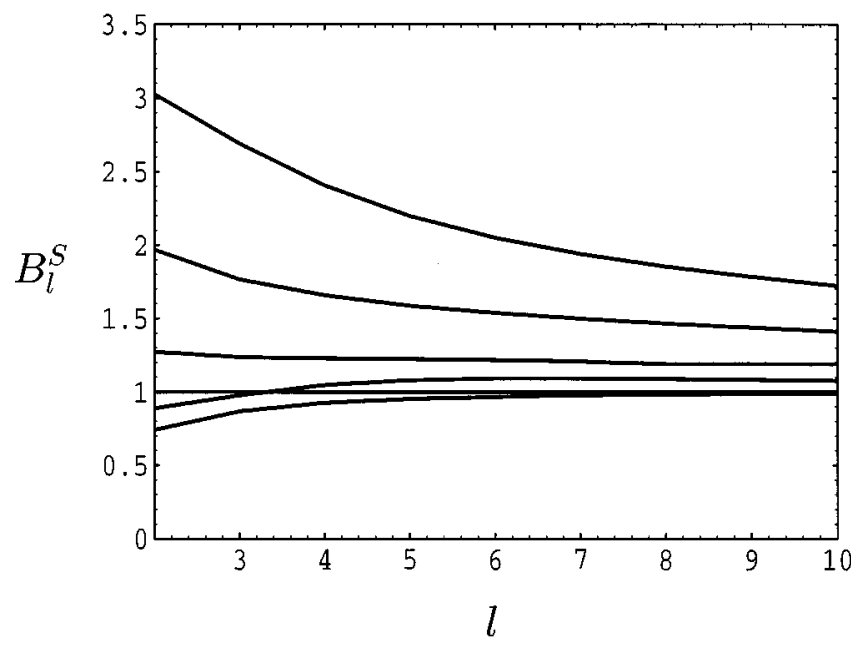

FIG. 2. The first 10 multipoles of the angular power spectrum associated with the scale-invariant density perturbations, $B_{l}^{S}$, for $\Omega_{0}=0.3,0.4,0.5,0.6,0.8$, from top to bottom. The straight line at $B_{l}^{S}=1$ gives the normalization, for $\Omega_{0}=1$; see Eq. (54).

$$
\left\langle\mathcal{R}_{q} \mathcal{R}_{q^{\prime}}\right\rangle=\frac{2 \pi^{2} \mathcal{P}_{\mathcal{R}}(q)}{q\left(1+q^{2}\right)} \delta\left(q-q^{\prime}\right)
$$

In the case of single-bubble open inflation models it can be written as [22]

$$
\begin{aligned}
& \mathcal{P}_{\mathcal{R}}(q)=A_{S}^{2} f(q), \\
& A_{S}^{2}=\frac{\kappa^{2}}{2}\left(\frac{H_{T}}{2 \pi}\right)^{2} \frac{1}{\epsilon},
\end{aligned}
$$

The function $f(q)$ depends on features of the single-bubble open inflation model [22]:

$$
f(q)=\operatorname{coth} \pi q-\frac{z^{2} \cos \tilde{q}+2 q z \sin \tilde{q}}{\left(4 q^{2}+z^{2}\right) \sinh \pi q},
$$

where $\tilde{q}=q \ln [(1+x) /(1-x)]$ and

$$
\begin{gathered}
x=\left(1-R^{2} H_{T}^{2}\right)^{1 / 2}=\Delta\left(1+\Delta^{2}\right)^{-1 / 2}, \\
z=\left(1-R^{2} H_{T}^{2}\right)^{1 / 2}-\left(1-R^{2} H_{F}^{2}\right)^{1 / 2}=2 b\left(1+\Delta^{2}\right)^{-1 / 2},
\end{gathered}
$$

see Eq. (3). The function $f(q)$ is linear at small $q$, and approaches a constant value $f(q)=1$ at $q \geqslant 2$. We will study its dependence on the parameters $a$ and $b$ in Appendix B. For scalar perturbations, the effect of $f(q)$ in the power spectrum $C_{l}^{S}$ is not very important. Therefore, the tilt of the scalar perturbation spectrum is very approximately given by Eq. (31). However, for a scale-invariant spectrum $n_{S}=1$ in an open universe, $l(l+1) C_{l}^{S}$ is no longer constant:

$$
l(l+1) C_{l}^{S}=\frac{2 \pi}{25} A_{S}^{2} B_{l}^{S}\left(\Omega_{0}\right),
$$

where $B_{l}^{S}$ is a function of $\Omega_{0}$, which approaches a constant value at $l \sim 20$, where the calculation breaks down as the power spectrum rises to the first acoustic peak. We have plotted this function in Fig. 2, for a scale-invariant spectrum and for various values of $\Omega_{0}$. For a tilted $n=1.15$ scalar spectrum see Ref. [30].

From the four year Cosmic Background Explorer (COBE) maps [3], the overall amplitude and tilt of the CMB power spectrum have been determined with some accuracy $[47,48]$ :

$$
\begin{gathered}
{\left[\frac{l(l+1) C_{l}^{S}}{2 \pi}\right]^{1 / 2}=(1.03 \pm 0.07) \times 10^{-5},} \\
n_{S}=1.02 \pm 0.24,
\end{gathered}
$$

assuming that the observed temperature anisotropy on large scales is solely determined by the scalar contribution (54). This determines the scalar amplitude to be

$$
A_{S}=\frac{1}{\sqrt{\pi \epsilon}} \frac{H_{T}}{M_{\mathrm{Pl}}} \simeq 5 \times 10^{-5}
$$

from which we extract a useful relation

$$
H_{T} \simeq \sqrt{\epsilon} 10^{-4} \mathrm{M}_{\mathrm{Pl}}
$$

Note that a tensor contribution $R$ to the total CMB power spectrum would reduce the amplitude $A_{S}$ by a factor $(1+R)^{-1 / 2}$. We will assume as in Refs. $[47,9]$ that $R \ll 1$. As we will see, in open hybrid models this may not be a good approximation and we will have to take this into account.

\section{Tensor modes}

Let us now study the gravitational wave power spectrum in an open universe. For perturbations that reenter during the matter era, the growing mode solution of Eq. (23) is $h(\eta)=h G_{q}(\eta)$, where $h$ is the amplitude of the primordial gravitational wave perturbation, at Hubble-scale crossing during inflation and later on at reentry during the matter era, and

$$
G_{q}(\eta) \equiv 3 \frac{\sinh \eta \sin q \eta-2 q \cos q \eta(\cosh \eta-1)}{q\left(1+4 q^{2}\right)(\cosh \eta-1)^{2}}
$$

is normalized so that $G_{q}(0)=1$ at the last scattering surface. Here $q^{2}=k^{2}-3$ for the tensor mode. The induced temperature fluctuation amplitude is then given by Eq. (35), where $Q_{r r}$ is the $r r$ component of the open universe tensor harmonic:

$$
Q_{r r}(r)=\left[\frac{(l-1) l(l+1)(l+2)}{2 q^{2}\left(1+q^{2}\right)}\right]^{1 / 2} \frac{\Pi_{q l}(r)}{\sinh ^{2} r} .
$$

The corresponding tensor power spectrum can be written as [39]

$$
C_{l}^{T}=(l-1) l(l+1)(l+2) \int_{0}^{\infty} \frac{d q \pi^{2} \mathcal{P}_{g}(q)}{8 q^{3}\left(1+q^{2}\right)^{2}} W_{q l}^{2},
$$

where $q^{2}=k^{2}-3$ and $W_{q l}$ is given by

$$
W_{q l}=\int_{0}^{\eta_{0}} d r G_{q}^{\prime}\left(\eta_{0}-r\right) \frac{\Pi_{q l}(r)}{\sinh ^{2} r} \text {. }
$$




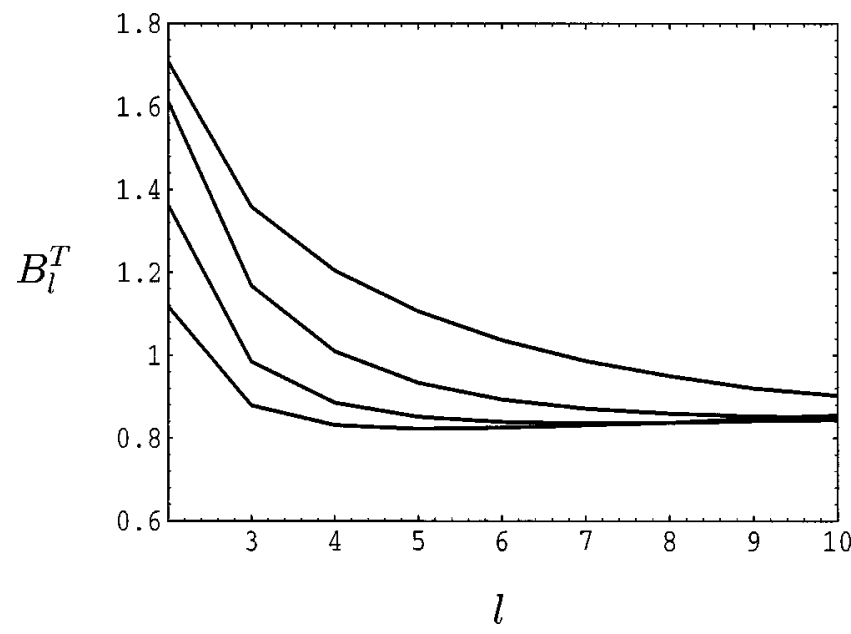

FIG. 3. The first 10 multipoles of the angular power spectrum associated with the scale-invariant gravitational wave perturbations, $B_{l}^{T}$, for $\Omega_{0}=0.4,0.6,0.8,1.0$, from top to bottom, and $f(q)$ $=\tanh (\pi q / 2)$.

Here $\mathcal{P}_{g}(q)$ is the primordial spectrum of open universe gravitational wave perturbations, defined by

$$
\left\langle h_{q} h_{q^{\prime}}\right\rangle=\frac{2 \pi^{2} \mathcal{P}_{g}(q)}{q\left(1+q^{2}\right)} \delta\left(q-q^{\prime}\right) .
$$

In the case of single-bubble open inflation models it can be written as [32]

$$
\begin{aligned}
& \mathcal{P}_{g}(q)=A_{T}^{2} f(q), \\
& A_{T}^{2}=8 \kappa^{2}\left(\frac{H_{T}}{2 \pi}\right)^{2} .
\end{aligned}
$$

Here $f(q)$ is the same function (51), which is linear at small $q$ and thus avoids the infrared divergence at $q=0$ found in Ref. [49], but quickly approaches $f(q)=1$ for $q \geqslant 2$. The effect of $f(q)$ on the tensor power spectrum could become important only for the first few multipoles. The best situation occurs in the limit $a \ll 1$ and $b \simeq 1$ [see Eq. (4)] in which this function becomes $f(q) \simeq \tanh (\pi q / 2)$. However, much larger contributions are possible for smaller values of $b$; see Appendix B.

The corresponding tilt of the gravitational wave spectrum can again be defined, at large $q$, as in Eq. (41). For a scaleinvariant spectrum, $n_{T}=0$, we can numerically integrate Eq. (61) to give

$$
l(l+1) C_{l}^{T}=\frac{\pi}{36}\left(1+\frac{48 \pi^{2}}{385}\right) A_{T}^{2} B_{l}^{T}\left(\Omega_{0}\right),
$$

where $B_{l}^{T}$ is a function of $\Omega_{0}$, which approaches a constant value for large multipoles, $l \sim 30$, beyond which the calculation breaks down and the tensor contribution decays very rapidly [33]. We have plotted this function in Fig. 3, for a scale-invariant spectrum and for various values of $\Omega_{0}$, and $f(q)=\tanh (\pi q / 2)$.

It is now interesting to compute the ratio of tensor to scalar components of the open universe power spectrum, as a function of $\Omega_{0}$. In the limit $l \gg 1$, the ratio $R_{l}=C_{l}^{T} / C_{l}^{S}$ ap-

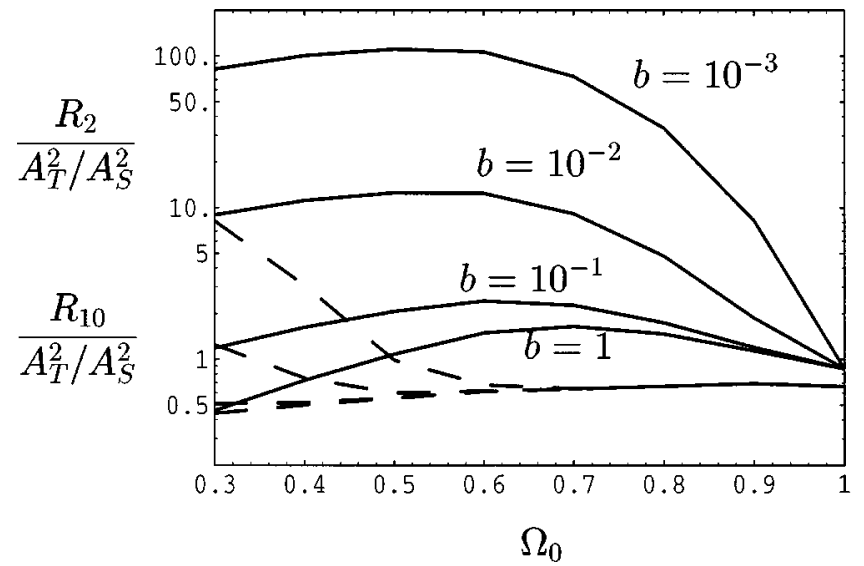

FIG. 4. The ratio of tensor to scalar components of the angular power spectrum for the quadrupole (continuous lines), $R_{2}=C_{2}^{T} / C_{2}^{S}$, and the tenth multipole (dashed lines), $R_{10}=C_{10}^{T} / C_{10}^{S}$, as a function of $\Omega_{0}$, for $a=0$ and $b=10^{-3}, 10^{-2}, 10^{-1}, 1$, from top to bottom. The ratio $R_{2}$ increases very quickly for very small values of the parameter $b$ in the range $\Omega_{0} \sim 0.3-0.8$, while $R_{10}$ only grows at small $\Omega_{0} \lesssim 0.5$.

proaches the flat space limit (43). However, for small multipoles, the difference with respect to the $\Omega_{0}=1$ value could be large, as we can see from Fig. 4, where the corresponding ratio for the quadrupole and the tenth multipole is shown. ${ }^{2}$ We have also shown the dependence of the ratio $R_{2}$ with the tunneling parameter $b$. It is clear from Fig. 4 that values of $b \lesssim 10^{-2}$ are not allowed, unless $A_{T}^{2} / A_{S}^{2}=16 \epsilon$ is very small indeed. However, the ratio $R_{10}$ does not grow as quickly as $R_{2}$ and for most values of $\Omega_{0}$ is below 1 .

The ratio $R_{2} /\left(A_{T}^{2} / A_{S}^{2}\right)=B_{2}^{T}\left(\Omega_{0}\right) / B_{2}^{S}\left(\Omega_{0}\right)$ depends very strongly on $b$. For $b=1$ we can approximate it by $1 \pm 0.5$ in the range of interest; see Fig. 4. The condition $R_{2}<1$ then imposes the constraint

$$
\epsilon<\frac{1}{16}
$$

However, for smaller values of $b$, the constraint is much stronger and also depends on the particular value of $\Omega_{0}$. For example, for $b=10^{-2}$ and $\Omega_{0} \sim 0.3-0.7$, the condition is ten times stronger, $\epsilon<1 / 160$, while for $b=10^{-3}$ in the same range, the condition is 100 stronger, $\epsilon<1 / 1600$. This means that the flat space relation (43) between the ratio of tensor to scalar contributions and the tensor spectral index is no longer valid. In open inflation models the relation is now a function of cosmological and model parameters:

$$
R_{l}=\frac{C_{l}^{T}}{C_{l}^{S}} \simeq f_{l}\left(\Omega_{0}, a, b\right) 8\left|n_{T}\right|\left[1-1.3\left(n_{S}-1\right)\right] .
$$

In the ideal case in which the gravitational wave perturbation can be disentangled from the scalar component in future precise observations of the CMB power spectrum, one might be

\footnotetext{
${ }^{2}$ The peak in the ratio $R_{2}$ is due to an accidental cancellation in the scalar power spectrum between the intrinsic and integrated Sachs-Wolfe effect [see Eq. (47)], which occurs at $\Omega_{0} \simeq 0.786$, where the distance to the last scattering surface coincides with the curvature scale, i.e., $\eta_{0} \simeq 1$.
} 
able to test this relation for a given value of $\Omega_{0}$. This would then constitute a check on the tunneling parameter $b$. Such prospects are, however, very bleak from measurements of the temperature power spectrum alone, with the next generation of satellites; see, e.g., $[50,6]$. At most one can expect to impose constraints on the parameters of the model from the absence of a significant gravitational wave contribution to the CMB. However, taking into account also the polarization power spectrum, together with the temperature data, one expects to do much better; see Refs. [46,8] for the case of flat models. We hope similar conclusions can be reached in the context of open models, and CMB observations may be able to check the generalized consistency relation (68) with some accuracy [51].

\section{Supercurvature mode}

Apart from the continuum of subcurvature modes, in open inflation we also have a contribution to the microwave background anisotropies coming from a discrete supercurvature mode, $k^{2}=0$, which appears in the spectrum of the inflaton field in open de Sitter when $m_{F}^{2}<2 H_{F}^{2}$ in the false vacuum [22]. The metric perturbation for this supercurvature mode is $[26,27]$

$$
A_{\mathrm{SC}}^{2} \simeq \frac{\kappa^{2}}{2}\left(\frac{H_{F}^{2}}{2 \pi}\right)^{2} \frac{1}{\epsilon}=A_{S}^{2} \frac{H_{F}^{2}}{H_{T}^{2}},
$$

where $A_{S}^{2}$ is given by Eq. (50).

In Refs. [27,30], we computed the corresponding power spectrum as a function of multipole number $l$. We are only interested here in the overall contribution of the supercurvature mode to the total power spectrum, relative to that of the scalar modes.

The ratio $C_{2}^{\mathrm{SC}} / C_{2}^{S}$ is somewhat dependent on $\Omega_{0}$ (see Fig. 5) but we can approximate it very roughly by $10^{-2} A_{\mathrm{SC}}^{2} / A_{S}^{2}$ in the range of interest. The condition $C_{2}^{\mathrm{SC}}<C_{2}^{S}$ then imposes a mild constraint on the rates of expansion in the false and true vacuum:

$$
H_{F}^{2}<10^{2} H_{T}^{2} .
$$

For a given value of $\Omega_{0}$ this constraint can be determined with greater precision; see Fig. 5.

\section{Bubble wall mode}

Apart from the continuum of subcurvature modes and the discrete supercurvature mode, we expect also a contribution from the bubble wall fluctuations [24,25,37]. These fluctuations contribute as a transverse traceless curvature perturbation mode with $k^{2}=-3$, which nevertheless behaves as a homogeneous random field; see Refs. [23,52]. In Ref. [32] it was argued that the bubble wall mode is actually not a discrete mode, once we include the gravitational backreaction. However, its effect on the CMB anisotropies can still be computed as if it were a discrete mode with $k^{2}=-3$. The constraints on open inflation models from the absence of this mode's contribution to the CMB do not change much under this assumption.

The curvature perturbation amplitude for this bubble wall mode can be computed from $[25,22]$
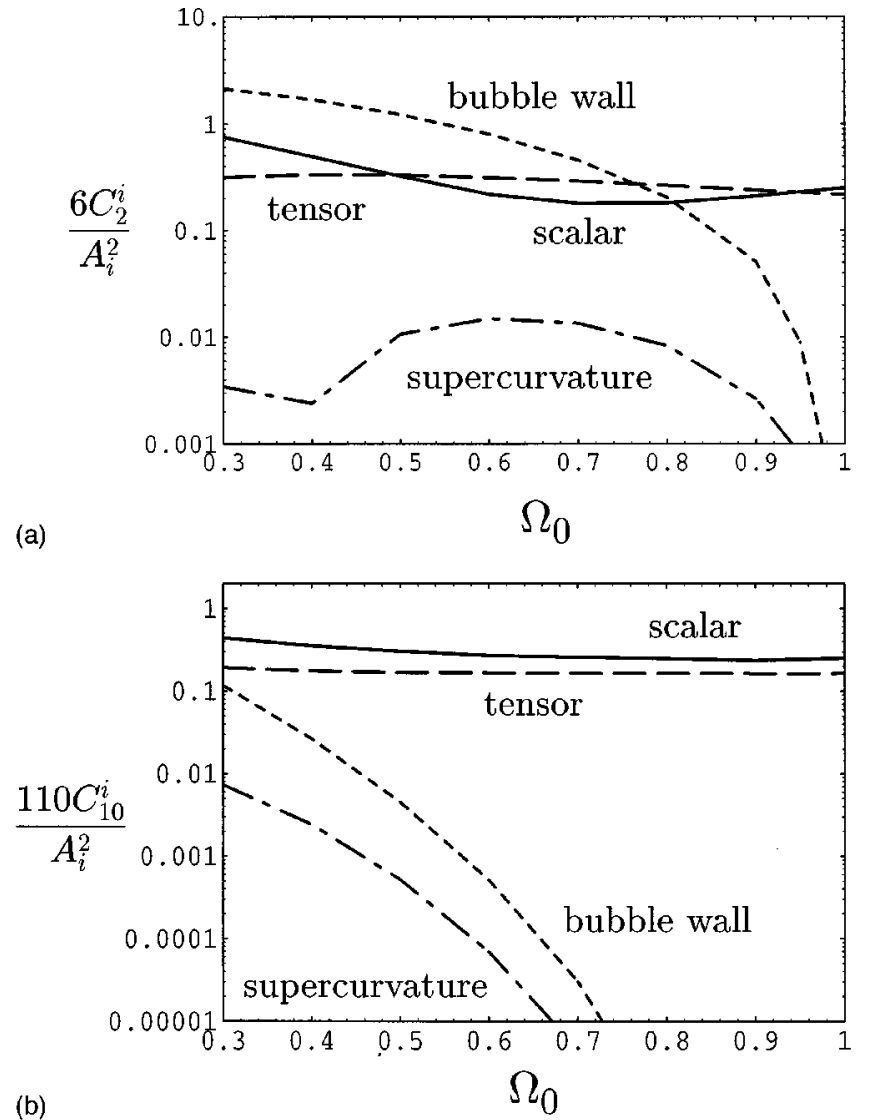

FIG. 5. The quadrupole and tenth multipole of the angular power spectrum, normalized to the corresponding metric perturbation, for the scalar (continuous lines), tensor (dashed lines), bubble wall (dotted lines) and supercurvature modes (dotted-dashed lines), as a function of $\Omega_{0}$. While the continuum of scalar and tensor modes do not change much as we approach $\Omega_{0}=1$, the supercurvature and bubble wall modes decrease exponentially.

$$
A_{W}^{2}=\frac{\kappa^{2}}{2}\left(\frac{H_{T}^{2}}{2 \pi}\right)^{2} \frac{1}{z}=A_{S}^{2} \frac{\epsilon}{z},
$$

where $z$ is given in Eq. (53) and $A_{S}^{2}$ is the scalar amplitude of Eq. (50).

In Refs. [27,30], we computed the corresponding power spectrum as a function of multipole number $l$. We are only interested here in the overall contribution of the bubble wall mode to the total power spectrum, relative to that of the scalar modes.

The ratio $C_{2}^{W} / C_{2}^{S}$ is slightly dependent with $\Omega_{0}$ (see Fig. 5) but we can approximate it very roughly by $A_{W}^{2} / A_{S}^{2}$ in the range of interest. The condition $C_{2}^{W}<C_{2}^{S}$ then imposes the constraint

$$
\epsilon<z
$$

For a given value of $\Omega_{0}$ this constraint can be determined with greater precision; see Fig. 5 .

\section{MODELS OF OPEN INFLATION}

We have obtained generic constraints on open inflation models from the individual components of the CMB power spectra. It is now necessary to explore particular models in 
order to test their viability. In this section we will concentrate on two concrete open inflation models that have definite predictions for the complete power spectra of temperature anisotropies of the CMB: the induced gravity open inflation model of Ref. [13] and the tilted open hybrid inflation model of Ref. [30].

\section{A. Induced gravity open inflation}

In Ref. [27] we computed only the scalar power spectrum of CMB anisotropies for the induced gravity open model, without including the gravitational wave contribution since its primordial spectrum was not yet known. The tensor primordial spectrum has recently, and simultaneously, been obtained by several groups; see Refs. [32]. We will show that this model of open inflation is compatible with CMB observations and contributes with negligible gravitational wave perturbations.

In this model, the tunneling occurs due to the field $\sigma$, with potential

$$
U(\sigma)=U_{F}+\frac{\lambda^{\prime}}{4} \sigma^{2}\left(\sigma-\sigma_{0}\right)^{2}-\mu U_{0}\left(\sigma / \sigma_{0}\right)^{4},
$$

where $\sigma_{0}=M^{\prime} \sqrt{2 / \lambda^{\prime}}$ corresponds to the true vacuum and $U_{0}=M^{\prime 4} / 16 \lambda^{\prime}$ is the value of the potential at its maximum, with $\mu \ll 1$ for the thin wall approximation to be valid.

The inflaton field $\varphi$ is nonminimally coupled to gravity, with coupling $\xi$, and possesses a symmetry breaking potential, $V(\varphi)=\lambda\left(\varphi^{2}-\nu^{2}\right)^{2} / 8$; see Ref. [13,27]. The false vacuum energy density of the sigma field, $U_{F}$, determines a stable fixed point for $\varphi$, in the false vacuum [27]:

$$
\varphi_{\mathrm{st}}^{2}=\nu^{2}\left(1+\frac{8 U_{F}}{\lambda \nu^{4}}\right) \equiv \nu^{2}(1+\alpha) .
$$

The corresponding false vacuum rate of expansion in the Einstein frame is given by $H_{F}^{2}=\left(\lambda \nu^{2} / 24 \xi\right) \alpha /(1+\alpha)$. After tunneling, the $\sigma$ field lies in its true vacuum at $U\left(\sigma_{0}\right) \simeq U_{F}-\mu U_{0}=0$. In this case, the field $\phi$ is no longer trapped and starts to evolve down its potential, driving inflation. Immediately after tunneling, the rate of expansion in the true vacuum, inside the bubble, is related to that in the false vacuum by [27]

$$
H_{T}^{2}=H_{F}^{2} \frac{\alpha}{1+\alpha} .
$$

Therefore, for parameter $\alpha \geqslant 1$, the rates of expansion are very similar. This will suppress the contribution of the supercurvature mode to the CMB power spectrum; see Eq. (70).

The amplitude and the tilt of the scalar perturbation spectrum are determined by the slow-roll parameters soon after tunneling:

$$
\begin{gathered}
\epsilon=\frac{8 \xi}{1+6 \xi} \frac{1}{\alpha^{2}}, \\
\eta=\frac{8 \xi}{1+6 \xi} \frac{1-\alpha}{\alpha^{2}} .
\end{gathered}
$$

The spectral tilt of scalar and tensor perturbations is always negative [27]:

$$
\begin{gathered}
n_{S}-1=-\frac{8 \xi}{1+6 \xi} \frac{2(2+\alpha)}{\alpha^{2}}, \\
n_{T}=-\frac{8 \xi}{1+6 \xi} \frac{2}{\alpha^{2}} .
\end{gathered}
$$

Let us now study the contribution from fluctuations of the bubble wall, Eq. (71). For that purpose we have to compute the tunneling parameters $a$ and $b$. From Eqs. (4) and (75) we see that $a b=1 / 4 \alpha$, while $b$ is given by

$$
b=\frac{\kappa^{2} S_{1}}{4 H_{T}}=\frac{2 \pi}{3 \lambda^{\prime}} \frac{M^{\prime}}{H_{T}}\left(\frac{M^{\prime}}{M_{\mathrm{Pl}}}\right)^{2},
$$

where $S_{1}=M^{\prime 3} / 3 \lambda^{\prime}$ is the contribution to the bounce action coming from the bubble wall, see Eq. (2), and we have used the COBE normalization (58). We will consider here large values of $M^{\prime} / H_{T}$ and thus prevent a supercurvature mode of the $\sigma$ field; see Ref. [19]. In this limit, $a \ll 1$ and

$$
z \simeq \frac{2 b}{\left(1+b^{2}\right)^{1 / 2}}
$$

The condition (72) on the bubble wall contribution to the CMB then determines

$$
b>\epsilon / 2,
$$

which is not very difficult to satisfy in the models of Ref. [27], where $\epsilon$ is small.

Furthermore, in the limit $a \ll 1$, the smallest contribution of the tensor modes to the CMB anisotropies comes from the parameter $b \simeq 1$, where $f(q) \simeq \tanh (\pi q / 2)$; see Appendix B. Induced gravity models determine the parameter $a b=1 / 4 \alpha$, and it is always possible to choose $a$ so that $b \simeq 1$. In this case, the constraint on the tensor amplitude comes from the quadrupole, which requires $R_{2}\left(\Omega_{0}\right)<1$; see Fig. 4. For most $\Omega_{0}$ in the range of interest, this constraint is satisfied provided $\epsilon \lesssim 1 / 16$. Again, this is not difficult to satisfy in the induced gravity open inflation model [13,27].

For example, in the case $\xi \ll 1$, we can have $8 \xi=1 / 200$, $\alpha=1$, together with $a=1 / 4, b=1, \lambda^{\prime}=0.1$, and $M^{\prime}$ $=10^{3} H_{T}=7 \times 10^{-3} M_{\mathrm{Pl}}$. This gives $\epsilon=1 / 200, H_{F}^{2} / H_{T}^{2}=2$, and $z \simeq 1.25$, which satisfies all constraints.

On the other hand, in the case $\xi \gg 1$, we have $\alpha=85$, together with $a=1 / 340, \quad b=1, \quad \lambda^{\prime}=0.1$, and $M^{\prime}=3$ $\times 10^{3} H_{T}=4 \times 10^{-3} M_{\mathrm{Pl}}$. This gives $\epsilon=2 \times 10^{-4}, H_{F}^{2} / H_{T}^{2}$ $\simeq 1$, and $z \simeq 1.41$, which again satisfies all constraints.

Induced gravity models are thus viable scenarios of open inflation, with small and negative spectral tilt and negligible contribution of gravitational waves to the CMB power spectrum.

\section{B. Open hybrid inflation}

Open hybrid inflation [30] was proposed recently in an attempt to produce a significantly tilted scalar spectrum in the context of open models. It is based on the hybrid inflation 
scenario [53], which has recently received some attention from the point of view of particle physics [54,55], together with a tunneling field which sets the initial conditions inside the bubble.

In this model there are three fields: the tunneling field $\sigma$, the inflaton field $\phi$, and the triggering field $\psi$. Quantum tunneling occurs in the $\sigma$ field due to its coupling to the $\phi$ field. When $\phi$ drops below a certain value, $\phi_{*}$, the true vacuum appears and there is an increasing probability that the $\sigma$ field will tunnel to it, creating a single bubble inside which the $\phi$ field will slow-roll down its potential driving inflation and producing the observed metric perturbations, until it drops below another scale, $\phi_{c}$, for which the triggering field $\psi$ acquires a negative mass and suffers a sudden phase transition which ends inflation. This second period is known as hybrid inflation; see Ref. [53].

The complete potential is the sum of the open model plus the hybrid model [30]:

$$
\begin{aligned}
V(\sigma, \phi, \psi)= & -\frac{M^{2} \psi^{2}}{2}+\frac{\lambda \psi^{4}}{4}+\frac{M^{4}}{4 \lambda} \exp \left(\frac{4 \pi \alpha \phi^{2}}{3 M_{\mathrm{Pl}}^{2}}\right)+\frac{M^{\prime 2} \sigma^{2}}{2} \\
& -\sqrt{\lambda^{\prime}} M^{\prime} \sigma^{3}+\frac{\lambda^{\prime} \sigma^{4}}{4}+\frac{\phi^{2}}{2}\left(g^{2} \psi^{2}+h^{2} \sigma^{2}\right)+\widetilde{V}_{0},
\end{aligned}
$$

where $\widetilde{V}_{0} \simeq M^{\prime 4} / \lambda^{\prime}$ has been added to make the effective potential vanish in the global minimum, $\phi=0$, $\psi=M / \sqrt{\lambda}, \sigma=(3+\sqrt{5}) M^{\prime} / 2 \sqrt{\lambda^{\prime}}$. Quantum tunneling occurs at $\phi=\phi_{*}=M^{\prime} / h$ and the phase transition that triggers the end of inflation occurs at $\phi=\phi_{c}=M / g$.

This model is rather constrained by the potential of the tunneling field, due to its coupling to the inflaton field. In particular, the vacuum energy of the $\sigma$ field cannot be much larger than that of the $\psi$ field, and its contribution to the effective mass of the $\phi$ field should also be suppressed:

$$
\begin{gathered}
\frac{h^{2} M^{\prime 2}}{\lambda^{\prime}} \leqslant \frac{2 \pi \alpha M^{4}}{3 \lambda M_{\mathrm{Pl}}^{2}}, \\
\frac{M^{\prime 4}}{\lambda^{\prime}} \leqslant \frac{M^{4}}{\lambda} .
\end{gathered}
$$

These constraints might prove to be too strong once we include the gravitational wave CMB power spectrum.

The rate of expansion during inflation is dominated by the false vacuum energy of the $\psi$ field:

$$
H^{2}=\frac{2 \pi}{3 \lambda} \frac{M^{4}}{M_{\mathrm{Pl}}^{2}} .
$$

The number of $e$-folds is

$$
N=\frac{3}{\alpha} \ln \left(\frac{g M^{\prime}}{h M}\right)=55 .
$$

The slow-roll parameters become

$$
\begin{gathered}
\epsilon=\frac{4 \pi \alpha^{2} \phi^{2}}{9 M_{\mathrm{Pl}}^{2}}, \\
\eta=\frac{\alpha}{3}\left(1+\frac{8 \pi \alpha \phi^{2}}{3 M_{\mathrm{Pl}}^{2}}\right),
\end{gathered}
$$

from which the tilt of the scalar and tensor spectra can be computed as

$$
\begin{gathered}
n_{S}-1=\frac{2 \alpha}{3}-2 \epsilon, \\
n_{T}=-2 \epsilon .
\end{gathered}
$$

On the other hand, the bubble parameter (2) is here $S_{1}=2 \sqrt{2} M^{\prime 3} / 3 \lambda^{\prime}$, which substituted into Eq. (4) gives

$$
b=2 \sqrt{2} \frac{H}{M^{\prime}}\left(\frac{\lambda M^{\prime 4}}{\lambda^{\prime} M^{4}}\right) .
$$

This parameter must always be approximately less than one in our model, since $M^{\prime} \geqslant H$ in order to prevent HawkingMoss tunneling [12] and/or a large supercurvature mode perturbation [19], and $M^{\prime 4} / \lambda^{\prime} \leqslant M^{4} / \lambda$ for $\psi$-vacuum domination during inflation. This means that gravitational waves could give an important contribution to the CMB anisotropies in these models.

Consider, for instance, the particular values $\alpha=0.25, g=0.2, \lambda=0.1$, and $M=1.5 \times 10^{-3} M_{\mathrm{Pl}}$ for the tilted hybrid model, and $h=10^{-3}, \lambda^{\prime}=0.01$, and $M^{\prime}=M / 2$ for the tunneling field. In that case, Eqs. (90)-(92) give

$$
\begin{gathered}
n_{S} \simeq 1.15, \quad n_{T} \simeq-0.02, \\
b \simeq 0.03, \quad \epsilon \simeq 0.01 .
\end{gathered}
$$

Note that the spectral tilts, $n_{S}$ and $n_{T}$, are significantly different from their scale-invariant values $n_{S}=1, n_{T}=0$. This is a generic feature of these models. We also have a large contribution to the power spectrum coming from the bubble wall fluctuations [see Eq. (72)] as well as from tensor perturbations, $R_{2} \simeq 0.65$ (for small $\Omega_{0}$, see Fig. 4), which could be potentially dangerous. However, the quadrupole is by far the largest multipole and could be hidden in the cosmic variance uncertainty for small multipoles [7]:

$$
\sigma_{l}=\left[\frac{2}{(2 l+1) f_{\text {sky }}}\right]^{1 / 2} C_{l},
$$

where $f_{\text {sky }}$ is the fraction of the sky covered by the particular experiment. For the quadrupole $l=2$ and a typical fraction $f_{\text {sky }}=1 / 2$, we find that the estimated error $\sigma_{2}$ is of the same order as $C_{2}$ and thus a tensor contribution with $R_{2}<1$ could be hidden in the CMB temperature maps. On the other hand, by the time we reach the tenth multipole, the ratio $R_{10}$ has decreased considerably (see Fig. 4) and therefore these parameters are still allowed by observations.

Open hybrid models are thus viable models of inflation with the special property of producing a positively tilted 


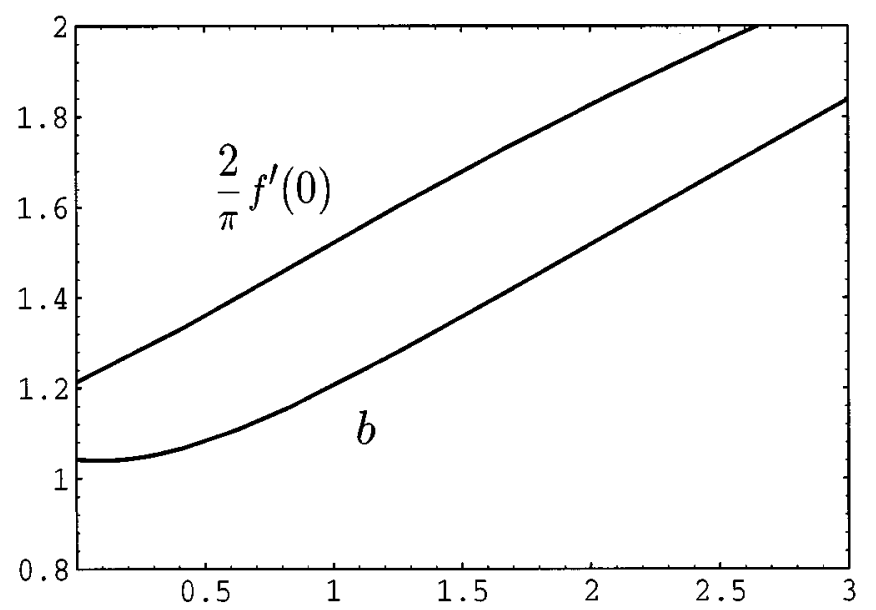

(a)

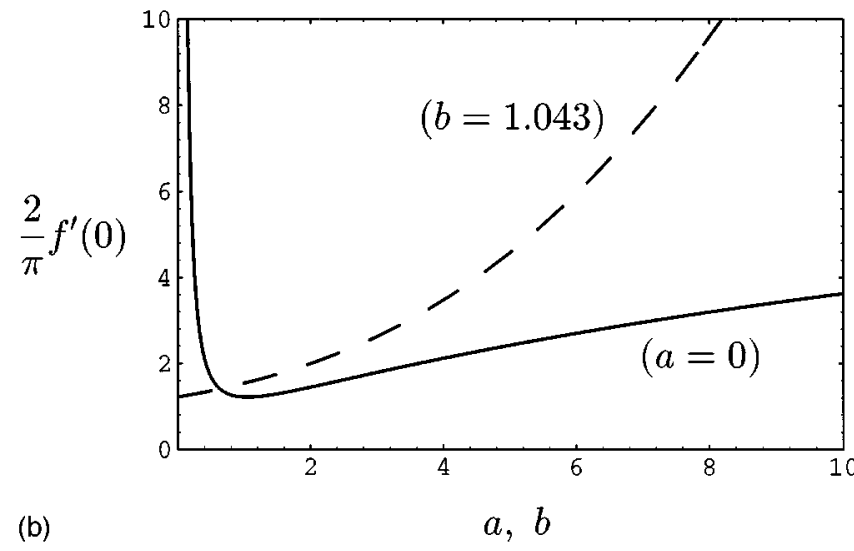

FIG. 6. The top panel shows the minimum slope of $f(q)$ and the corresponding value of $b$ as a function of the parameter $a$. They approach asymptotically the values $f^{\prime}(0)=1.214 \pi / 2$ and $b=1.043$, respectively. The lower panel shows the sharp increase in the slope of $f(q)$ at small values of the parameter $b$, for $a=0$ (continuous line) and at large values of $a$, for $b=1.043$ (dashed line).

spectrum of density perturbations, which might help the agreement with observations of large scale structure [28] and CMB anisotropies [29].

\section{CONCLUSIONS}

In the near future, observations of the microwave background will determine with better than $1 \%$ accuracy whether we live in an open universe or not. It is therefore crucial to know whether inflation can be made compatible with such a universe. Single-bubble open inflation models provide a natural scenario for understanding the large scale homogeneity and isotropy. Furthermore, inflationary models generically predict a nearly scale invariant spectrum of density and gravitational wave perturbations, which could be responsible for the observed CMB temperature anisotropies. Future observations could then determine whether inflationary models are compatible with the observed features of the CMB power spectrum. For that purpose it is necessary to know the predicted power spectrum from inflation with great accuracy. Open models have a more complicated primordial spectrum of perturbations, with extra discrete modes and possibly large tensor anisotropies. In order to constrain those models

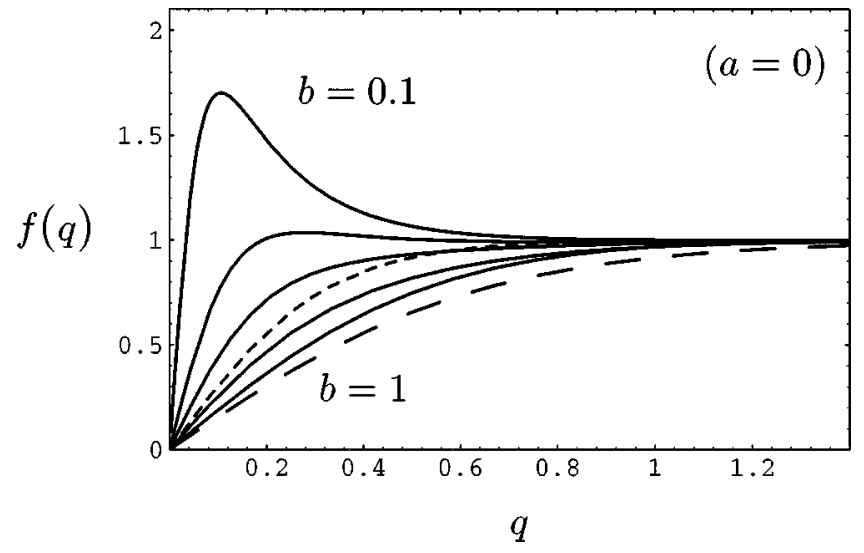

FIG. 7. The spectral function $f(q)$ for $a=0$ and various values of $b=0.1,0.2,0.3,0.5,1.0$, from top to bottom (continuous lines). As we decrease $b$ the function develops a bump at small $q$ which becomes very pronounced for very small values of $b$, increasing significantly the gravitational wave contribution to the CMB power spectrum. The dashed line corresponds to $f(q)=\tanh \pi q / 2$ and the dotted line to $f(q)=\tanh \pi q$.

we have to compute the full spectrum for a large range of parameters.

In this paper we have computed the large scale angular power spectrum of temperature fluctuations in the CMB induced by gravitational wave perturbations in the context of the single-bubble open inflation models. We have then studied the dependence of the ratio $R$ of tensor to scalar components with the value of $\Omega_{0}$ and the tunneling parameter $2 \pi G S_{1} / H$. We have shown that $R$ increases very quickly for very small values of this parameter. The flat-space consistency relation between the ratio $R$ and the tensor spectral index $n_{T}$ is now a more complicated relation, which mainly depends on the tunneling parameter $2 \pi G S_{1} / H$. In the ideal case in which the gravitational wave perturbation can be disentangled from the scalar component in future precise observations of the $\mathrm{CMB}$ power spectrum, one might be able to test this relation for a given value of $\Omega_{0}$. Such prospects are, however, very bleak from measurements of the temperature power spectrum alone, with the recently approved new generation of satellites; see, e.g., [50,7]. At most one can expect to impose constraints on the parameters of the model from the absence of a significant gravitational wave contribution to the CMB. However, taking also into account the CMB polarization power spectrum, together with the temperature power spectrum, one expects to do much better; see Refs. $[46,8]$ for the case of flat models. We hope similar conclusions can be reached in the context of open models, and CMB observations may be able to test the generalized consistency relation with some accuracy [51].

We have found a set of constraints from scalar, tensor, supercurvature, and bubble wall modes' contribution to the CMB anisotropies that the parameters of a general open inflation model should satisfy in order to agree with observations. We have applied such constraints to the induced gravity and open hybrid inflation models and found a range of parameters which make them compatible with present observations. In the future we might be able to determine these parameters with greater precision.

Note added. While writing this paper, we noticed the 
work of Sasaki et al. [56], where similar conclusions were reached.

\section{ACKNOWLEDGMENTS}

It is a pleasure to thank Andrei Linde for constant encouragement and in particular for his comments on the best parameters for open hybrid models.

\section{APPENDIX A: OPEN UNIVERSE MODE FUNCTIONS}

The open universe scalar mode functions are discussed in Refs. [36,52]. The correctly normalized subcurvature scalar modes can be written as

$$
\Pi_{q l}(r)=N_{q l} \widetilde{\Pi}_{q l}(r)
$$

with

$$
N_{q l}=\sqrt{\frac{2}{\pi}} \prod_{n=1}^{l}\left(n^{2}+q^{2}\right)^{-1 / 2}, \quad N_{q 0}=\sqrt{\frac{2}{\pi}},
$$

where the unnormalized modes $\widetilde{\Pi}_{q l}(r)$ can be generated from the first two

$$
\begin{gathered}
\widetilde{\Pi}_{q 0}(r)=\frac{\sin q r}{\sinh r}, \\
\widetilde{\Pi}_{q 1}(r)=\frac{\operatorname{coth} r \sin q r-q \cos q r}{\sinh r},
\end{gathered}
$$

through the recurrence relation

$$
\begin{aligned}
\widetilde{\Pi}_{q l}(r)= & (2 l-1) \operatorname{coth} r \widetilde{\Pi}_{q, l-1}(r)-\left[(l-1)^{2}+q^{2}\right] \\
& \times \widetilde{\Pi}_{q, l-2}(r) .
\end{aligned}
$$

In the limit $\Omega_{0} \rightarrow 1$, the scale factor becomes $a$ $\propto 2(\cosh \eta-1)=\sinh ^{2} \eta \rightarrow \eta^{2}$; the eigenvalues of the Laplacian $q^{2} \rightarrow k^{2}$, and the scalar eigenfunctions become

$$
\Pi_{q l}(r) \rightarrow \sqrt{\frac{2}{\pi}} k j_{l}(k r) .
$$

On the other hand, the correctly normalized radial component of the tensor modes can be written as [36]

$$
Q_{r r}(r)=\left[\frac{(l-1) l(l+1)(l+2)}{2 q^{2}\left(1+q^{2}\right)}\right]^{1 / 2} \frac{\Pi_{q l}(r)}{\sinh ^{2} r},
$$

where $\Pi_{q l}(r)$ is the scalar mode (A1). In the limit $\Omega_{0} \rightarrow 1$, this tensor mode becomes $Q_{r r}$ in Eq. (36), as expected, while $G_{q}(r) \rightarrow G_{k}(r)$.

\section{APPENDIX B: DEPENDENCE OF THE GRAVITATIONAL WAVE SPECTRUM ON THE TUNNELING PARAMETERS}

In this Appendix we will study the behavior of the function $f(q)$, Eq. (51), in the spectrum of gravitational wave perturbations. This function behaves as $f(q) \simeq \tanh (\pi q / 2)$ in a certain well-defined limit. However, for arbitrary values of the tunneling parameters (4), it can take a very different shape. For certain parameters, it increases very quickly at small $q$, thus giving a large contribution to the gravitational wave power spectrum of CMB anisotropies. It is therefore important to study its behavior at small $q$, where $f(q)$ is linear. The slope at the origin is given by

$$
f^{\prime}(0)=\frac{\pi}{2}\left[1+\frac{1+\Delta^{2}+\left[2 b \ln \left(\Delta+\sqrt{1+\Delta^{2}}\right)-\sqrt{1+\Delta^{2}}\right]^{2}}{\pi^{2} b^{2}}\right],
$$

where $\Delta$ is defined in Eq. (4). For $b \simeq 1$ we recover the limiting function, $f(q) \simeq \tanh (\pi q / 2)$. For all other values, the slope (B1) is larger and so is the contribution of the tensor modes to the CMB anisotropies. The best situation corresponds to those values of $a$ and $b$ for which (B1) is as small as possible.

Let us now study the behavior of the slope (B1) with various tunneling parameters. In Fig. 6 we show the minimum possible slope and the corresponding value of $b$ for a given value of $a$. One can see that the smallest contribution to the CMB comes from $a=0$, and $b=1.043 \simeq 1$, for which the slope becomes $1.214 \pi / 2$. This gives a slightly larger contribution than the minimum function $\tanh (\pi q / 2)$. On the other hand, for $b<1$, the minimum slope increases very quickly, as we can see in Fig. 6.

We have shown various functions $f(q)$ in Fig. 7, for $a=0$ and $b=0.1-1$. The effect of a large $f(q)$ at small $q$ is an increased tensor contribution to the CMB. This effect is very important for small $\Omega_{0}$, as we can see in Fig. 4. In Ref. [33] this large effect at small values of the parameter $b$ was not realized since they assumed that the function $f(q)$ was bounded between $\tanh (\pi q)$ and $\tanh (\pi q / 2)$, what they called maximal and minimal tensor anisotropies. As one can see from Fig. 7, larger gravitational wave anisotropies are possible in certain classes of models.
[1] A. D. Linde, Particle Physics and Inflationary Cosmology (Harwood Academic, Chur, Switzerland, 1990).

[2] A. R. Liddle and D. H. Lyth, Phys. Rep. 231, 1 (1993).

[3] C. L. Bennett et al., Astrophys. J. 464, L1 (1996).

[4] C. Lineweaver, D. Barbosa, A. Blanchard, and J. Bartlett, astro-ph/9610133, astro-ph/9612146, 1996.

[5] MAP Home Page at http://map.gsfc.nasa.gov/ (1996).

[6] PLANCK (formerly COBRAS/SAMBA) Home Page at http:/
/astro.estec.esa.nl/SA-general/Projects/Cobras/cobras.html (1996).

[7] G. Jungman, M. Kamionkowski, A. Kosowsky, and D. N. Spergel, Phys. Rev. D 54, 1332 (1996).

[8] M. Zaldarriaga, D. Spergel, and U. Seljak, astro-ph/9702157, 1997.

[9] J. R. Bond, G. Efstathiou, and M. Tegmark, astro-ph/9702100, 1997. 
[10] J. R. Gott, Nature (London) 295, 304 (1982); J. R. Gott III and T. S. Statler, Phys. Lett. 136B, 157 (1984).

[11] M. Bucher, A. S. Goldhaber, and N. Turok, Phys. Rev. D 52, 3314 (1995); M. Bucher and N. Turok, ibid. 52, 5538 (1995); K. Yamamoto, M. Sasaki, and T. Tanaka, Astrophys. J. 455, 412 (1995).

[12] A. D. Linde, Phys. Lett. B 351, 99 (1995); A. D. Linde and A. Mezhlumian, Phys. Rev. D 52, 6789 (1995).

[13] A. Green and A. R. Liddle, Phys. Rev. D 55, 609 (1997).

[14] W. L. Freedman et al., Nature (London) 371, 757 (1994); N. R. Tanvir et al., ibid. 377, 27 (1995).

[15] M. Bolte and C. J. Hogan, Nature (London) 376, 399 (1995).

[16] M. W. Feast and R. M. Catchpole (unpublished); I. N. Reid, astro-ph/9704078, 1997.

[17] A. Dekel, astro-ph/9705033, 1997.

[18] A. R. Liddle, D. H. Lyth, R. K. Schaefer, Q. Shafi, and P. T. P. Viana, Mon. Not. R. Astron. Soc. 281, 531 (1996).

[19] M. Sasaki, T. Tanaka, and K. Yamamoto, Phys. Rev. D 51, 2979 (1995).

[20] D. H. Lyth and A. Woszczyna, Phys. Rev. D 52, 3338 (1995).

[21] J. García-Bellido, A. R. Liddle, D. H. Lyth, and D. Wands, Phys. Rev. D 52, 6750 (1995).

[22] K. Yamamoto, M. Sasaki, and T. Tanaka, Phys. Rev. D 54, 5031 (1996).

[23] T. Hamazaki, M. Sasaki, T. Tanaka, and K. Yamamoto, Phys. Rev. D 53, 2045 (1996).

[24] J. García-Bellido, Phys. Rev. D 54, 2473 (1996).

[25] J. Garriga, Phys. Rev. D 54, 4764 (1996).

[26] M. Sasaki and T. Tanaka, Phys. Rev. D 54, R4705 (1996).

[27] J. García-Bellido and A. R. Liddle, Phys. Rev. D 55, 4603 (1997).

[28] A. R. Liddle, D. H. Lyth, D. Roberts, and P. T. P. Viana, Mon. Not. R. Astron. Soc. 278, 644 (1996).

[29] M. White and J. Silk, Phys. Rev. Lett. 77, 4704 (1996).

[30] J. García-Bellido and A. Linde, Phys. Lett. B 398, 18 (1997); Phys. Rev. D 55, 7480 (1997).

[31] J. García-Bellido and D. Wands, Phys. Rev. D 54, 7181 (1996); A. Linde and V. Mukhanov, ibid. 56, 535 (1997).

[32] T. Tanaka and M. Sasaki, Prog. Theor. Phys. 97, 243 (1997); M. Bucher and J. D. Cohn, Phys. Rev. D 55, 7461 (1997).

[33] W. Hu and M. White, astro-ph/9701210, 1997.
[34] S. Parke, Phys. Lett. 121B, 313 (1983).

[35] J. M. Bardeen, Phys. Rev. D 22, 1882 (1980); V. F. Mukhanov, H. A. Feldman, and R. H. Brandenberger, Phys. Rep. 215, 203 (1992).

[36] E. R. Harrison, Rev. Mod. Phys. 39, 862 (1967); L. F. Abbott and R. K. Schaefer, Astrophys. J. 308, 546 (1986).

[37] J. D. Cohn, Phys. Rev. D 54, 7215 (1996).

[38] R. K. Sachs and A. M. Wolfe, Astrophys. J. 147, 73 (1967).

[39] A. A. Starobinsky, Pis'ma Astron. Zh. 11, 323 (1985) [Sov. Astron. Lett. 11, 133 (1985)].

[40] W. Hu, N. Sugiyama, and J. Silk, astro-ph/9604166, 1996.

[41] M. Kamionkowski, D. N. Spergel, and N. Sugiyama, Astrophys. J. 426, L57 (1994); W. Hu and N. Sugiyama, Phys. Rev. D 51, 2599 (1995).

[42] D. H. Lyth, Phys. Rev. D 31, 1792 (1985).

[43] J. R. Bond and G. Efstathiou, Mon. Not. R. Astron. Soc. 226, 655 (1987).

[44] E. F. Bunn, D. Scott, and M. White, Astrophys. J. 441, L9 (1995).

[45] L. F. Abbott and M. B. Wise, Phys. Lett. 135B, 279 (1984).

[46] U. Seljak and M. Zaldarriaga, Astrophys. J. 469, 437 (1996); D. Spergel and M. Zaldarriaga, astro-ph/9705182, 1997.

[47] J. R. Bond, in Cosmology and Large Scale Structure, Les Houches Summer School Course LX, edited by R. Schaeffer (Elsevier Science Press, Amsterdam, 1996).

[48] E. F. Bunn, A. R. Liddle, and M. White, Phys. Rev. D 54, 5917 (1996).

[49] B. Allen and R. Caldwell, preprint WISC-MILW-94-TH-21 (unpublished).

[50] L. Knox and M. S. Turner, Phys. Rev. Lett. 73, 3347 (1994).

[51] M. Kamionkowski (private communication).

[52] J. García-Bellido, A. R. Liddle, D. H. Lyth, and D. Wands, Phys. Rev. D 55, 4596 (1997).

[53] A. D. Linde, Phys. Lett. B 259, 38 (1991); Phys. Rev. D 49, 748 (1994).

[54] L. Randall, M. Soljačić, and A. H. Guth, Nucl. Phys. B472, 377 (1996).

[55] J. García-Bellido, A. D. Linde, and D. Wands, Phys. Rev. D 54, 6040 (1996).

[56] M. Sasaki, T. Tanaka, and Y. Yakushige, Phys. Rev. D 56, 616 (1997). 